\title{
KIF5B-RET Fusion gene may coincide oncogenic mutations of EGFR or KRAS gene in lung adenocarcinomas
}

Jeong-Oh Kim', Jieun Lee ${ }^{2}$, Jung-Young Shin ${ }^{1}$, Ji-Eun Oh', Chan-Kwon Jung ${ }^{3}$, Jae Kil Park', Sook-Whan Sung ${ }^{4}$, Sang-Ju Bae ${ }^{5}$, Hyun-Jung Min ${ }^{5}$, Dowon Kim ${ }^{6}$, Jae Yong Park ${ }^{7}$ and Jin-Hyoung Kang ${ }^{2^{*}}$

\begin{abstract}
Background: The KIF5B-RET rearrangement is detected with the frequency of $1 \sim 2 \%$ in 'triple marker'-negative lung adenocarcinomas, i.e., EGFR, KRAS and EML4-ALK wild type. These mutational changes are known to be mutually exclusive, but the co-existence of ALK rearrangement with activating mutations of EGFR is rarely found.

Methods: We examined the KIF5B-RET fusion gene in frozen tissues from 154 surgically resected lung tumors using RT-PCR with direct sequencing and the mutation status of EGFR and KRAS genes using PNA clamping. We tested KIF5B-RET translocation in Formalin Fixed Paraffin Embedded using fluorescence in situ hybridization. We also measured RET mRNA and protein expression by RT-PCR and immunohistochemistry, respectively.

Results: The existence of KIF5B-RET fusion gene was identified in 9 patients. The mean age was 67.2 and M: F ratio 4:5. Of 9 patients, 3 patients harbored wild type of EGFR and KRAS gene. However, KIF5B-RET fusion gene coincided with EGFR or KRAS mutation in 6 patients. These six pts were also positive for both RET break-apart probes (23.9\%) and KIF5B-RET fusion (44.4\%). However, there were no correlations between RET mRNA and protein expression in the KIF5B-RET-positive patients. The median disease free survival and overall survival were 23.9 months and 29.5 months, respectively.

Conclusions: Taken together, our data suggest one-step screening platform for KIF5B-RET as well as EGFR, K-RAS, ALK oncogenic mutations be necessary for lung adenocarcinoma patients because EGFR or KRAS mutation are not infrequently found in KIF5B-RET-positive patients.
\end{abstract}

Keywords: Lung adenocarcinoma, KIF5B-RET fusion gene, Fluorescence in situ hybridization

\section{Background}

Over the past 10 years, great advances in molecular biology have enabled the discovery of various driver mutations of lung adenocarcinomas, including the epidermal growth factor receptor (EGFR), ALK, AKT1, BRAF, HER2, KRAS, MET, PIK3CA, RET, ROS1. The proliferation of tumor cells that originates from a single oncogene mutation is termed as an 'oncogene addiction'. Specific blocking of an oncogene effectively inhibits the growth of tumor cells, leading to the development of targeted drugs in oncology [1-3].

\footnotetext{
* Correspondence: jinkang@catholic.ac.kr

${ }^{2}$ Department of Medical Oncology, Seoul St. Mary's Hospital, The Catholic University of Korea, 222 Banpo-daero Seocho-gu, Seoul, Republic of Korea Full list of author information is available at the end of the article
}

In 2012, we first identified the existence of the Kinesin family member 5B (KIF5B)-RET fusion gene in a young male patient with lung adenocarcinoma using wholegenome and transcriptome sequencing analysis. This gene rearrangement, involving the fusion of exon 16 from $K I F 5 B$ and exon 12 from $R E T$ can strongly influence oncogenesis [4]. The KIF5B-RET fusion gene is rarely found in Asians or never smokers, and is mutually exclusive to other oncogenic mutations including those in the EGFR, KRAS, BRAF, and ERBB2 gene, or EML4-ALK gene. The KIF5B-RET fusion gene has been reported to occur in 1-2 \% of lung adenocarcinomas; however, adenocarcinomas of other anatomical sites lack this mutation. Moreover, the correlation of KIF5B-RET fusion gene with smoking is still ambiguous [5]. 
The transplantation of the K15:R12 variant of KIF5BRET fusion gene-transformed cells into nude mice resulted in the development of the tumor in vivo, indicating the fusion gene's oncogenic potential [6,7]. In the ongoing NCT001639508 study, cabozantinib demonstrated disease control activity for patients harboring RET gene rearrangements, including the KIF5B-RET fusion gene [8]. These encouraging preliminary results suggest that the $R E T$ fusion gene may be a promising druggable target [8,9]. Although the aforementioned driver mutations were accepted to be mutually exclusive, several recent studies have reported the co-existence of $A L K$ rearrangement with EGFR or KRAS mutations. Moreover, other investigators have shown that the sequential administration of the EGFR tyrosine kinase inhibitor (TKI) and ALK inhibitors could induce stable disease for the patients harboring concomitant EGFR mutation and $A L K$ rearrangement [10]. These results imply that patients harboring concomitant oncogenic driver mutations may show different biologic behaviors and clinical outcomes to the targeted therapy.

In this study, we examined the frequency of the KIF5B-RET fusion gene in Korean NSCLC patients and whether this gene rearrangement coincides with other oncogenic drivers.

\section{Methods}

\section{Patients}

Between February 2009 and June 2013, we enrolled patients who were diagnosed with pulmonary adenocarcinomas at Seoul St. Mary's Hospital and Korea Lung Tissue Bank of the Infrastructure Project of Basic Science of the Ministry of Education, Science, and Technology. All patients received surgical pulmonary resection that was pathologically confirmed as an adenocarcinoma by a senior pathologist. All tumor samples were immediately frozen after surgical resection, and stored at $-80{ }^{\circ} \mathrm{C}$ until the experiment. Remaining tumor samples were stored as formalin-fixed paraffin-embedded (FFPE) tissues.

Written informed consent was obtained from all participants, and this study was approved by the Institutional Review Board of Seoul St Mary's Hospital (approval No. KC13SISI0040) and Guro Hospital, Korea University (approval No. KU Guro Gene Bank.2013-015).

Reverse transcription polymerase chain reaction (RT-PCR) for the KIF5B-RET fusion gene and RET mRNA expression

Total RNA were extracted from frozen tissues as per manufacture's protocols using ZR-Duet DNA/RNA Miniprep kit (Zymoresearch, Irvine, CA). RNA concentrations were determined using the Nano Drop ND-1000 spectrophotometer (Nano Drop Technologies Inc., Rockland, DE). After the synthesis of cDNA using the Maxime RT Premix (INTRON Biotechnology Inc, Korea), we designed as previously reported [4]. The sequences for the RET gene's kinase domain were used to design the forward primer: 5'-GAA GGC GAA TTT GGA AAA GT-3', and the reverse primer: 5' -ATACTG CAT CCC CTG TGA GA-3'. The reaction conditions were as follows: 40 cycles of $95^{\circ} \mathrm{C}$ for $10 \mathrm{sec}, 58{ }^{\circ} \mathrm{C}$ for $40 \mathrm{sec}$ and $72{ }^{\circ} \mathrm{C}$ for $7 \mathrm{sec}$, with a final extension for $10 \mathrm{~min}$ at $72{ }^{\circ} \mathrm{C}$. Glyceraldehyde 3phosphate dehydrogenase (GAPDH) was used as internal reference gene.

\section{PNA Clamp ${ }^{\mathrm{TM}}$ EGFR and KRAS mutation analysis}

Genomic DNA was determined using the Nano Drop ND-1000 Spectrophotometer. Mutational analysis for EGFR and KRAS was performed using the CFX96 RealTime PCR Detection System (Bio-Rad, Philadelphia, PA) with the PNA clamp ${ }^{\mathrm{TM}}$ EGFR and KRAS mutation detection kit (Panagene, Inc., Daejeon, Korea). All reactions performed as previously reported [2].

\section{Analysis of the KIF5B-RET fusion gene using FISH}

To detect the KIF5B-RET fusion gene in tumor tissues using FISH, $4 \mu$ m-thick FFPE sections were used; FFPE slides were deparaffinized and rehydrated, and were pretreated with citrate buffer (Cellay Inc., Cambridge, MA) for 15 minutes at $96-98{ }^{\circ} \mathrm{C}$, and then immersed in pepsin solution for 10 minutes at $37{ }^{\circ} \mathrm{C}$. The $\mathrm{FISH}$ probe was denatured for $5 \mathrm{~min}$ at $80{ }^{\circ} \mathrm{C}$, subsequently the slides were incubated for 20 hours at $37^{\circ} \mathrm{C}$ overnight.

The ZytoLight ${ }^{\bullet}$ SPEC RET Dual Color Break Apart Probe set (Zytovision Inc. Bremerhaven, Germany), KIF5B-RET SY Translocation FISH probe (Abnova, Taipei, Taiwan) and FISH detection kit were applied to the slides. After the post-hybridization washing, 4, 6-diamidino-2phenylindole were used for counter staining. The stained slides were assessed using a Carl Zeiss microscope with the tissue FISH analysis module using the Metafer software (MetaSystems, GmbH Altlussheim, Germany).

\section{Interpretation of immunohistochemical staining}

Immunohistochemical staining was performed on $4 \mu \mathrm{m}$ thick FFPE tumor tissue sections. The slides were deparaffinized, and antigen retrieval was performed in a steam cooker for 15 minutes in citrate buffer (0.01 M, pH 6.0). The sections were incubated overnight at $4{ }^{\circ} \mathrm{C}$ with primary antibodies for anti-RET (Rabbit monoclonal, 1:500 dilution, Epitomics, California, USA) and anti-ALK (Rabbit monoclonal, 1:250 dilution, Cell Signaling Technology, Danvers, MA, USA). After washing, signal was amplified by incubating the section with secondary antibody (Polink2 plus HRP Rabbit DAB kit; GBI,Mukilteo,WA, USA), for 10 minutes. The following rabbit monoclonal antibodies were used for immunohistochemical staining. Finally, the reaction was visualized with a 3,3'-diaminobenzidine as the chromogens. Slides were counterstained with hematoxylin before mounting. The levels of RET and ALK expression 
were scored on the basis of the staining intensity of tumor cells, and the relative proportion of positively stained cells among total tumor cells. The tumor cell staining intensity was graded as follows: 0 , absent; 1 , weak (light brown); 2 , moderate (brown); and 3, strong (dark brown).

\section{Statistical analysis}

Overall survival was calculated from the date of clinical diagnosis of lung adenocarcinoma to the death of patient, or patient's last follow-up date. Disease-free survival was calculated from the date of surgical resection to the date of disease recurrence, which was confirmed by imaging studies including chest CT scans. The statistical correlations between the results of immunohistochemistry for RET and FISH analysis for the KIF5B-RET gene rearrangement was analyzed using Student's t tests.

The frequency of KIF5B-RET fusion and RET mRNA expression was compared with paired t-test. All statistical analyses were performed with SPSS (ver. 18.0; SPSS, Chicago, IL, USA).

\section{Results}

\section{Baseline characteristic}

Among 154 patients, 83 patients were male (53.9\%), with no gender predominance. The median age was 62 years (range, 25-85 years). One hundred and fifteen patients $(74.4 \%)$ had an eastern cooperative oncology group performance status of $0,96(62.3 \%)$ were classified with stage I disease, and 45 patients (29.3\%) with stage II or III. Ninety-four patients $(61.0 \%)$ had moderately- differentiated adenocarcinomas (Table 1).

\section{Distribution of the KIF5B-RET fusion gene, and EGFR and KRAS mutations}

The expression of the KIF5B-RET fusion gene was analyzed in 154 archival tumor tissues. The mRNA was extracted from fresh frozen tumor tissue, and amplified by RT-PCR. The primers were designed based on the KIF5B exon 15, 16, and RET exon 12 (K15, 16, R12). We observed the amplified KIF5B-RET fusion gene in 9 patients (5.8\%) (Fig. 1a). Next, we confirmed the KIF5B-RET variant 16/12 by direct sequencing in all patients (Fig. 1b). In addition, we conducted direct sequencing and PNA clamping to examine whether the patients harbored EGFR or KRAS mutations. PNA clamping analysis revealed that 5 of 9 patients harbored an activating EGFR mutation, 2 had a microdeletion in exon 19, and 3 harbored missense mutations in exon 21 (L858R). For 5 patients, the EGFR mutation status was confirmed with direct sequencing; equivalent results were obtained, except for one patient with a microdeletion in exon 19, where a weak mutation peak was observed. Among the 9 patients, 1 patient (11.1\%) harbored a KRAS codon 12 mutation, which was analyzed by direct sequencing and PNA clamping
Table 1 Basic Characteristics of Patient's

\begin{tabular}{ll}
\hline Patient's Characteristics & No. (\%) \\
\hline No. of patients & 154 \\
Median age(range) & $62(25-85)$ \\
Sex & \\
Male & $83(53.9 \%)$ \\
Female & $71(46.1 \%)$ \\
Smoking & \\
Never smoker & $80(51.9 \%)$ \\
Ever smoker & $74(48.1 \%)$ \\
ECOG & \\
0 & $115(74.4 \%)$ \\
1 & $39(25.6 \%)$ \\
Initial stage & \\
I & $96(62.3 \%)$ \\
II & $24(15.6 \%)$ \\
III & $21(13.7 \%)$ \\
IV & $13(8.4 \%)$ \\
Differentiation & \\
Well differentiated & \\
Moderate differentiated & $43(27.9 \%)$ \\
\hline
\end{tabular}

(Fig. 2). The remaining 3 patients (33.3\%) were positive for the KIF5B-RET fusion gene exclusively, and harbored no EGFR and KRAS mutations, or $A L K$ rearrangement.

\section{The detection of KIF5B-RET fusion gene by FISH}

FISH analysis was performed to confirm the existence of the KIF5B-RET fusion gene at the DNA level in 9 samples with KIF5B-RET mRNA, as identified by RT-PCR. We counted 100 interphase nuclei within each tumor specimen in $100 \mathrm{X}$ high power fields under fluorescence microscopy. Tumor tissue with $>15 \%$ split red and green signal in 100 interphase nuclei was defined as positive for the RET fusion gene (Fig. 3). In our study, the average frequency of the RET break-apart probes $23.9 \%$ (range, 14.8-37.8) and KIF5B-RET fusion 44.4 \% (range, 22.272.4)in 9 tumor tissues (Fig. 3G). The average FISH split and fusion signal for RT-PCR negative cases were $4 \%$ (range, 2.1-10) and $18.8 \%$ (range,14.4-23.9), respectively.

The correlation between KIF5B-RET fusion gene and RET mRNA expression, and ALK protein expression by immunohistochemistry

Total RNA was extracted from the fresh frozen tumor tissues, in which the presence of KIF5B-RET fusion gene was confirmed by both RT-PCR and FISH analysis. We analyzed the correlation between the frequencies of KIF5B-RET fusion by FISH and RET mRNA, or RET 

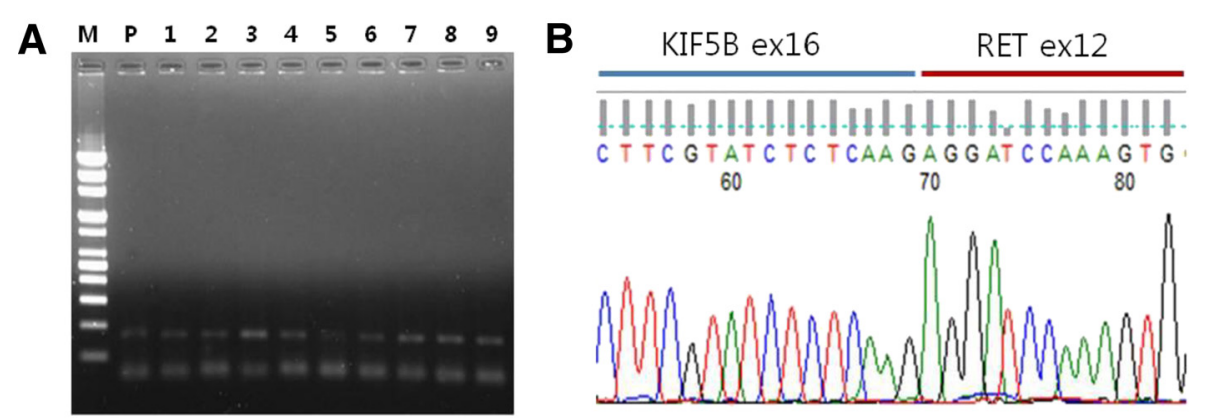

Fig. 1 KIF5B-RET fusion gene of RT-PCR and direct sequencing. a RT-PCR was analyzed using CDNA from 154 NSCLC patients. Samples observed the amplified KIF5B-RET fusion gene in 9 patients. b We confirmed that the KIF5B-RET varient $16 / 12$ by direct sequencing in all patients. Lane 1, size marker (M); lane 2, positive control (P); lane 3-12, number of patients

protein expression. In our analysis, the samples with RET protein expression tended to be positive for expression of the KIF5B-RET fusion gene by FISH, however the correlation was not statistically significant $(P=0.345)$ (Fig. 4a and Fig. 5b). Moreover, no statistical association could be determined between RET mRNA and expression of the KIF5B-RET fusion gene by FISH $(P=0.805)$ (Fig. 4b). Also, we analyzed ALK protein expression by immunohistochemical analysis using a monoclonal antibody specific for ALK fusion protein; no ALK expression could be detected in samples harboring the KIF5B-RET fusion gene (Fig. 5c).

\section{The association between the KIF5B-RET fusion gene and clinical variables}

We reviewed the clinical progress of 9 patients harboring the KIF5B-RET fusion gene. The median age was 67.2 years (range 56-82 years); 4 patients were male. Five patients were never smokers. The median relapse-free survival duration was 23.9 months (range 2.63-110.8 months), and median overall survival was 29.5 months (range 2.6-
114.5 months). All patients had histologically confirmed adenocarcinomas with acinar, lepidic, or papillary growth patterns. We detected the KRAS and EGFR mutation as well as the KIF5B-RET fusion gene in primary lung adenocarcinomas. We concluded that the lung tumor samples were based on the clinical history and the immunohistochemical data, which showed no expression of thyroid transcription factor (TTF-1) and napsin. In summary, 6 of 9 patients had either EGFR or KRAS mutations, and the remaining 3 patients were negative for both $E G F R$ and KRAS mutations (Table 2).

\section{Discussion}

The KIF5B-RET fusion gene is capable of inducing the abnormal proliferation and differentiation of tumor cells through the constitutional overexpression and activation of the RET proto-oncogene, ultimately leading to lung adenocarcinomas [4]. To date, the EGFR and KRAS gene mutations, and the EML4-ALK fusion gene have been identified as major oncogenic driver mutations of lung
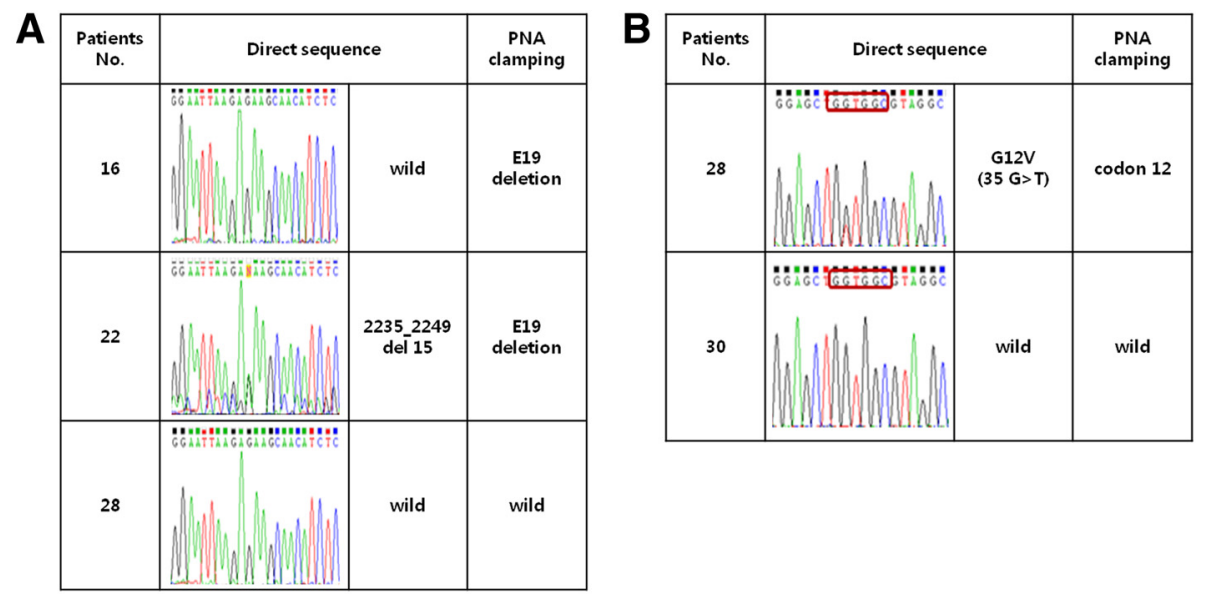

Fig. 2 EGFR and KRAS gene mutations in patients. EGFR (a) and KRAS (b) mutations were identified using the PNA clamping and direct sequencing method for 9 patients. PNA clamping analysis revealed similar results as those obtained from direct sequencing, one patient exhibited EGFR 19del on PNA clamping, but a weak mutant peak on direct sequencing 

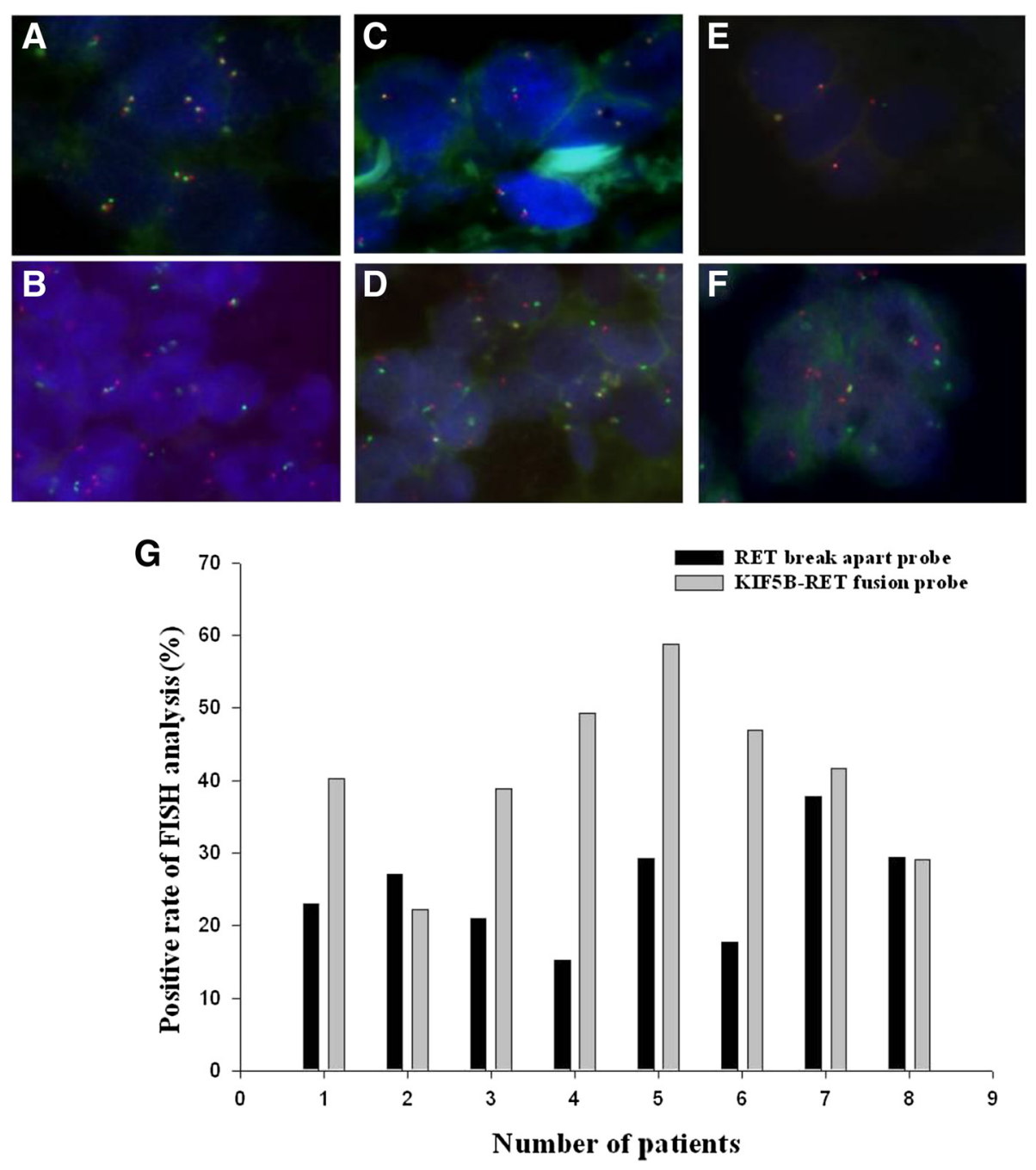

Fig. 3 FISH analysis for RET break apart probe (a, $\mathbf{c}, \mathbf{e})$ and KIF5B-RET fusion probe (b, d, $\mathbf{f})$. a and $\mathbf{b}$ show two types of FISH analysis for both wild type of EGFR and KRAS mutation status. $\mathbf{c}$ and $\mathbf{d}$ show FISH analysis for E19del mutation and wild type of KRAS mutation. e and $\mathbf{f}$ depict FISH analysis for wild type of EGFR mutation and codon 12 of KRAS mutation. $\mathbf{g}$ Comparing the frequency of RET break apart probe and KIF5B-RET fusion probe
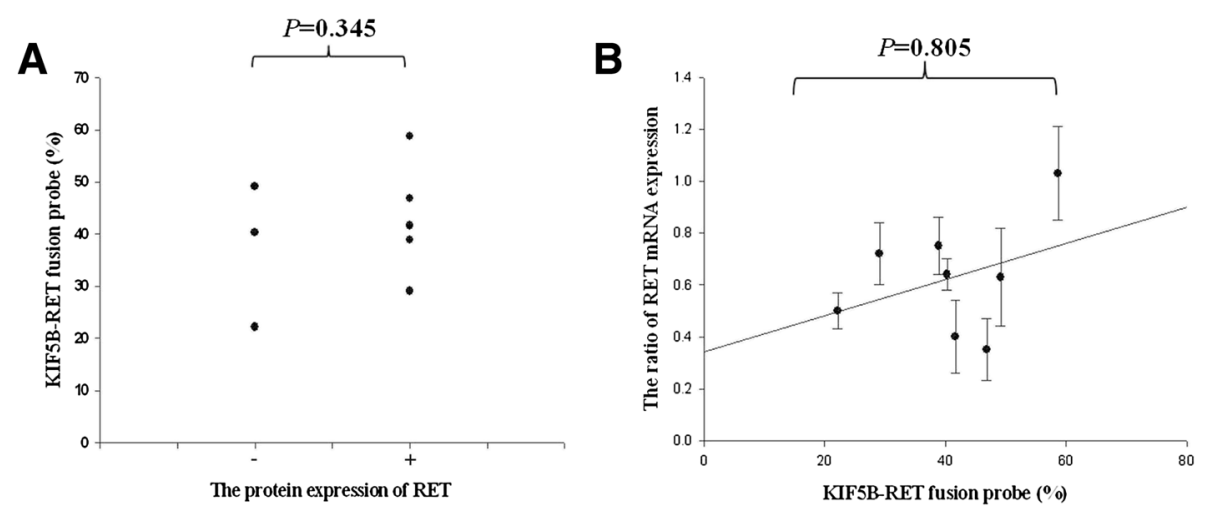

Fig. 4 The expression of RET mRNA and protein in KIF5B-RET fusion gene patients. a The patients having RET protein expression tended to be positive KIF5B-RET fusion gene by FISH, but with no statistical significance $(P=0.345)$. b FISH analysis and RET mRNA expression of the KIF5B-RET fusion gene was observed. There was no statistical association between RET mRNA and FISH $(P=0.805)$ 


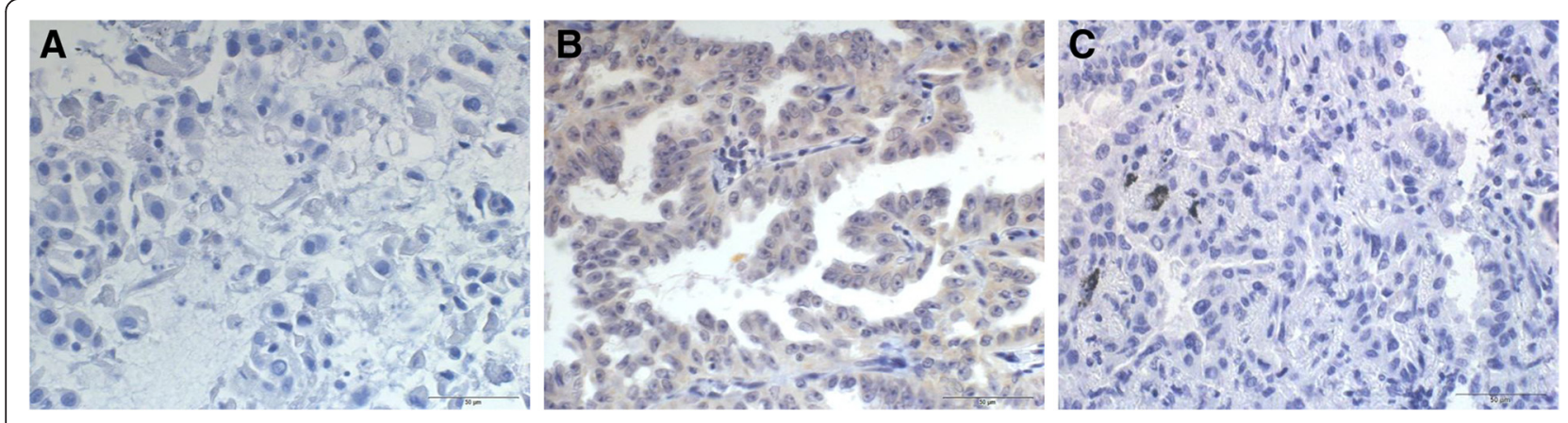

Fig. 5 The protein expression of RET and ALK (D5F3) using immunohisto-chemistry. Specimens (a, b) show negative and positive of RET IHC with RET rearrangement status of FISH analysis. Specimens (c) show negative ALK IHC with RET rearrangement status (original magnification, $\times 400$ )

adenocarcinomas. However, the molecular etiology of 40\% of adenocarcinomas remains to be discovered [3]. In the event that screening tests fail to detect driver mutations in advanced adenocarcinomas, palliative chemotherapy is the main treatment option.

Data from previous studies have consistently indicated that oncogenic driver mutations are mutually exclusive [3]. However, recent studies have shown that the $A L K$ fusion gene was found may occur concomitantly with the EGFR mutation. Yang et al. reported that the frequency of concomitant EGFR mutation and $A L K$ translocation was $1.3 \%$ in NSCLC [11]. Upon administering a specific tyrosine kinase inhibitor (TKI), dissimilar responses were observed in the patients harboring concomitant genomic alterations as compared to patients with single genetic alteration. Recently, Hirai et al. presented a case exhibiting poor response after the administration of an EGFR TKI in a lung adenocarcinoma patient harboring both, the KIF5BRET fusion gene and EGFR mutation [12]. In patients with concurrent EGFR mutation and $A L K$ rearrangement, the tumor response to certain TKIs may be inferior to historical data, depending on which genetic alteration is the major oncogenic driver. This hypothesis may explain the intrinsic resistance to EGFR TKIs in patients harboring concomitant genetic alterations $[11,12]$. We investigated the incidence of the KIF5B-RET fusion gene in Korean NSCLC patients, and attempted to identify the cases in which this fusion gene occurred concurrently with EGFR or KRAS mutations. We discovered KIF5B-RET fusion genes in 9 (5.8\%) out of 154 patients with lung adenocarcinoma, at a higher frequency than was reported previously [13]. Most prior studies had investigated the frequency of the KIF5B-RET fusion gene in an enriched cohort that was confirmed as triple negative by screening for the EGFR and $K-R A S$ mutations, and $E M L / A L K$ rearrangement $[13,14]$.

Conversely, in the present study, we screened all patients for the KIF5B-RET fusion genes using RT-PCR, irrespective of the status of EGFR and KRAS mutations, or EML/ALK fusion genes. The frequency of the KIF5B-RET fusion gene in patients lacking genetic alterations including $E G F R$, $K R A S$, and $A L K$ rearrangement was $1.9 \%$, similar to the results of the previous studies $[5,15]$. It is reasonable to assume that any discrepancies in the frequency of $K I F 5 B$ -

Table 2 Clinical and genetic characteristics of patients carrying KIF5B-RET fusion gene

\begin{tabular}{|c|c|c|c|c|c|c|c|c|c|c|}
\hline \multirow[t]{3}{*}{ No } & \multicolumn{3}{|c|}{ Clinical characteristics } & \multicolumn{7}{|c|}{ Genetic characteristics } \\
\hline & \multirow{2}{*}{$\begin{array}{l}\text { Sex/ } \\
\text { Age }\end{array}$} & \multirow[t]{2}{*}{ Smoking } & \multirow[t]{2}{*}{ Histology } & \multicolumn{2}{|c|}{ KIF5B-RET fusion gene } & \multirow[t]{2}{*}{ EGFR mutation } & \multirow{2}{*}{$\begin{array}{l}\text { KRAS } \\
\text { mutation }\end{array}$} & \multirow{2}{*}{$\begin{array}{l}\text { ALK } \\
\text { rearrangement }\end{array}$} & \multirow{2}{*}{$\begin{array}{l}\mathrm{OS}^{\mathrm{a}} \\
(\mathrm{mo})\end{array}$} & \multirow{2}{*}{$\begin{array}{l}\mathrm{DFS}^{\mathrm{b}} \\
(\mathrm{mo})\end{array}$} \\
\hline & & & & $\begin{array}{l}\text { Break apart } \\
\text { probe }(\%)\end{array}$ & $\begin{array}{l}\text { Fusion } \\
\text { probe (\%) }\end{array}$ & & & & & \\
\hline 1 & $F / 58$ & Never-smoker & Acinar & 23.0 & 40.3 & Wild & Wild & negative & 8.7 & 7.33 \\
\hline 2 & M/82 & current & Acinar & 27.1 & 22.2 & Wild & Wild & negative & 114.5 & 110.8 \\
\hline 3 & $M / 63$ & Ex-smoker & Acinar & 21.0 & 38.9 & Wild & Wild & negative & 53.4 & 15.23 \\
\hline 4 & $\mathrm{~F} / 71$ & Never-smoker & Acinar\& lepidic & 15.3 & 49.2 & E19del & Wild & negative & 9.6 & 9.63 \\
\hline 5 & $M / 56$ & Ex-smoker & Papillary & 29.2 & 58.7 & E19del & Wild & negative & 11.2 & 10.8 \\
\hline 6 & $\mathrm{~F} / 57$ & Never-smoker & Acinar\& lepidic & 17.8 & 46.9 & L858R or L861Q & Wild & negative & 2.6 & 2.63 \\
\hline 7 & $\mathrm{~F} / 74$ & Never-smoker & Acinar\& lepidic & 37.8 & 41.7 & L858R or L861Q & Wild & negative & 5.9 & 5.9 \\
\hline K15 & $F / 74$ & Never-smoker & Acinar & 14.8 & 72.4 & L858R & Wild & - & FU loss & FU loss \\
\hline 8 & $\mathrm{M} / 70$ & Ex-smoker & Papillary & 29.4 & 29.1 & Wild & Codon12 & negative & 30.1 & 29.37 \\
\hline
\end{tabular}

${ }^{\mathrm{a} O v e r a l l ~ s u r v i v a l ;}{ }^{\mathrm{b}}$ Disease free survival 
RET fusion gene may arise from dissimilarities in study populations. To date, seven variant isoforms of the KIF5B$R E T$ fusion gene have been identified; K15; R12 is the most common variant, accounting for $60-70 \%$ of KIF5B-RET gene variants $[5,16]$. In our study, we performed direct sequencing to verify the existence of variant K16; R12 and $\mathrm{K} 15$; R12, and then ascertained that K16; R12 was present in tumor tissues of all cases.

In addition, we carried out PNA clamping and direct sequencing to analyze whether the EGFR or KRAS mutation may coincide with KIF5B-RET-positive patients. One patient exhibited EGFR E19del on PNA clamping, but a weak mutant peak on direct sequencing [17]. We used next-generation sequencing to verify that PNA clamping was more sensitive and accurate. Currently, FISH is widely accepted to be the most useful tool for detection of structural genetic variations such as the $R E T$ rearrangement; it is a simpler, faster, and less expensive standard method, compared with whole-genome sequencing or transcriptome sequencing.

The results obtained from two types of FISH analysis were in agreement; as the cut-off level for positive cells in known potential $A L K$ gene rearrangement was used, the frequencies of RET rearrangements in the two FISH analyses may be higher than the cutoff of the $A L K$ gene analysis. In the case of $A L K$, the cut-off criteria was determined on the basis of an association study with the ALK inhibitor crizotinib [18]. We evaluated the RET protein expression induced by KIF5B-RET fusion by immunohistochemical analysis of paraffin-embedded tumor tissues with a RET C-peptide monoclonal antibody. Relatively weak staining intensity (1+) of RET protein was observed in most samples harboring the KIF5B-RET fusion gene as identified by RT-PCR and FISH analysis; however, the average intensity was higher than that of the patients with no KIF5B-RET fusion. A recent paper indicated that weak intensity of RET protein expression was observed in KIF5B-RET fusion-positive patients, which is in agreement with our observation [15]. In the present study, we observed a trend towards stronger intensity of RET protein expression concurrent with an increase in frequency of KIF5B-RET fusion gene $(P=0.345)$.

Conversely, no statistical significance was observed between the frequencies of KIF5B-RET fusion gene and RET mRNA expression $(P=0.805)$. These results imply that neither RET protein expression nor RET mRNA expression can successfully indicate the presence of the KIF5B-RET fusion. Structural alterations within the KIF5B-RET fusion protein may explain the lower reactivity to the conventional RET monoclonal antibody used in our study; thus, it is necessary to develop a specific antibody in order to assess the expression of KIF5B-RET fusion protein. Moreover, the correlations obtained with FISH analysis should be evaluated by measuring gene amplification, rather than mRNA expression of KIF5BRET fusion gene, as shown by Go et al. [19].

In contrast to previous reports, our results indicate that the KIF5B-RET fusion gene may coincide with EGFR or $K R A S$ mutations, albeit at a lower frequency, in lung adenocarcinomas. Further research is required to understand the functional molecular genetics of KIF5B-RET fusion gene, and to facilitate the development of a novel therapeutic drug targeting this fusion gene.

\section{Conclusions}

Our study demonstrated that the KIF5B-RET fusion gene was associated with EGFR or KRAS mutations, albeit at a low frequency. Therefore, screening for the EGFR and KRAS mutations, and ALK and KIF5B-RET translocations should be considered during initial diagnosis of lung adenocarcinomas. Moreover, clinical studies that combine RET inhibitors and EGFR TKIs with other targeted drugs are warranted for the lung adenocarcinoma patients harboring concurrent KIF5B-RET fusion gene and EGFR or KRAS mutations.

\section{Abbreviations \\ KIF5B-RET: Kinesin family member 5B; EGFR TKI: Epidermal growth factor receptor tyrosine kinase inhibitor; FFPE: Formalin-fixed paraffin-embedded; RT-PCR: Reverse transcription polymerase chain reaction; FISH: Fluorescence in situ hybridization; GAPDH: Glyceraldehyde 3-phosphate dehydrogenase.}

\section{Competing interests}

The authors declare that they have no competing interests.

\section{Authors' contributions}

JOK and JHK designed the study and drafted the manuscript. JOK,JYS,JEO carried out the experiments and analyzed of data. JEL,JHK, JKP,SWS, JYP; critical importance to the work described and collecting clinical data. CKJ; participated in interpreting staining slides and evaluation of the histology. SJB,HJM,DWK; participated in analysis of mutation and FISH. All authors read and approved the final manuscript.

\section{Acknowledgement}

This study was supported by a grant from the National R\&D Program for Cancer Control, Ministry of Health \& Welfare, Republic of Korea (1320030). We thank The KOREA University GURO Hospital of National Bank and their specimen donors for providing expression profile data.

The authors thank Young Seok Ju for review of this manuscript.

\section{Author details}

${ }^{1}$ Laboratory of Medical Oncology, Reaserch Institutes of Medical Science, The Catholic University of Korea, 222 Banpo-daero Seocho-gu, Seoul 137-701, Republic of Korea. 'Department of Medical Oncology, Seoul St. Mary's Hospital, The Catholic University of Korea, 222 Banpo-daero Seocho-gu, Seoul, Republic of Korea. ${ }^{3}$ Division of Pathology, Seoul St.Mary's hospital, The Catholic University of Korea, 222 Banpo-daero Seocho-gu, Seoul, Republic of Korea. ${ }^{4}$ Department of Thoracic and Cardiovascular Surgery, Seoul St.Mary's hospital, The Catholic University of Korea, 222 Banpo-daero Seocho-gu, Seoul, Republic of Korea. ${ }^{5}$ Mirax Ltd, 334-1 Jangan-gu Suwon-si, Gyeonggi-do, Republic of Korea. ${ }^{6}$ Panagene Inc, 816 Tamnip-dong Yuseong-gu, Daejeon, Republic of Korea. ${ }^{7}$ Department of Internal Medicine, Kyungpook National University School of Medicine, 130 Dongdeok-ro Jung-gu, Daegu, Republic of Korea.

Received: 15 April 2015 Accepted: 9 July 2015

Published online: 14 August 2015 


\section{References}

1. Manolo D, Federico C. K-Ras Mutations in Non-Small-Cell Lung Cancer: Prognostic and Predictive Value. ISRN Molecular Biol. 2012;837306:8.

2. Han HS, Lim SN, An JY, Lee KM, Choe KH, Lee KH, et al. Detection of EGFR Mutation Status in Lung adenocarcinoma Specimens with Different Proportions of Tumor Cells Using Two Methods of Differential Sensitivity. J Thorac Oncol. 2012;7(2):355-63.

3. William $P$, Nicolas $G$. New driver mutations in non-small-cell lung cancer. Lancet Oncol. 2011;12:175-80.

4. Ju YS, Lee WC, Shin JY, Lee SB, Thomas B, Won JK, et al. A transforming KIF5B and RET gene fusion in lung adenocarcinoma revealed from whole-genome and transcriptome sequencing. Genome Res. 2012;22(3):436-45

5. Kohno T, Ichikawa H, Totoki Y, Kazuki Y, Masaki H, Takao N, et al. KIF5B-RET fusions in lung adenocarcinoma. Nat Med. 2012;18(3):375-7.

6. Kengo T, Manabu S, Yuki T, Ritsuro S, Seiji S, Satoko H, et al. RET, ROS1 and ALK fusions lung cancer. Nat Med. 2012;18(3):378-81.

7. Lipson D, Capelletti M, Yelensky R, Geoff O, Alex P, Mirna J, et al. Identification of new ALK and RET gene fusions from colorectal and lung cancer biopsies. Nat Med. 2012;18(3):382-4.

8. Alexander D, Lu W, Adnan H, Yoshiyuki S, Dron LS, et al. Response to cabozantinib in patients with RET fusion-positive lung adenocarcinomas. Cancer Discov. 2013;3:630-5.

9. Thilo OG, Franziska AK, Strobel K, Hirschmann A, Aebi S, Diebold J. A Patient with Lung Adenocarcinoma and RET Fusion Treated with Vandetanib. J Thorac Oncol. 2013;8:5.

10. Akihiko M, Kumi S, Rintaro N, Masahiro S, Kazuhiro K, Seiji K, et al. Activity of EGFR-tyrosine kinase and ALK inhibitors for EML4-ALK-rearranged non-small-cell lung cancer harbored coexisting EGFR mutation. BMC Cancer. 2013;13:262.

11. Yang JJ, Zhang XC, Su J, Xu CR, Zhou Q, Tian HX, et al. Lung Cancers with Concomitant EGFR Mutations and ALK Rearrangements: Diverse Responses to EGFR-TKI and Crizotinib in Relation to Diverse Receptors Phosphorylation. Clin Cancer Res. 2014;20(5):1383-92.

12. Hirai F, Takenoyama M, Taguchi K, Ryo T, Eiko I, Gouji T, et al. Experience with erlotinib in lung adenocarcinoma harboring a coexisting KIF5B-RET fusion gene and EGFR mutation: report of a rare case. J Thorac Oncol. 2014;9(5):e37-9.

13. Yokota K, Sasaki H, Okuda K, Shimizu S, Shitara M, Hikosaka Y, et al. KIF5B/ RET fusion gene in surgically-treated adenocarcinoma of the lung. Oncol Rep. 2012;28(4):1187-92.

14. Wong DW, Leung EL, So KK, Tam IY, Sihoe AD, Cheng LC, et al. The EML4-ALK fusion gene is involved in various histologic types of lung cancers from nonsmokers with wild type EGFR and KRAS. Cancer. 2009;115:1723-33.

15. Hidefumi S, Shigeki S, Yoichi T, Masahiko M, Katsuhiro O, Keisuke Y, et al. RET expression and detection of KIF5B/RET gene rearrangements in Japanese lung cancer. Cancer Med. 2012;1(1):68-75.

16. Yoo SS, Jin G, Jung HJ, Hong MJ, Choi JE, Jeon HS, et al. RET Fusion Genes in Korean Non-Small Cell Lung Cancer. J Korean Med Sci. 2013;28:1555-8.

17. Kim HJ, Lee KY, Kim YC, Kim KS, Lee SY, Jang TW, et al. Detection and comparison of peptide nucleic acid-mediated real-time polymerase chain reaction clamping and direct gene sequencing for epidermal growth factor receptor mutations in patients with non-small cell lung cancer. Lung Cancer. 2012;75:321-5.

18. DRoss C, Mariana T, DeLee AM, Margaret S, Tara O, Xian L, et al. Correlations between the percentage of tumor cells showing an ALK gene rearrangement, ALK signal copy number and response to crizotinib therapy in ALK FISH positive non-small cell lung cancer. Cancer. 2012;118(18):4486-94.

19. Go HJ, Jung JY, Kang HW, Park IK, Kang CH, Lee JW, et al. Diagnostic method for the detection of KIF5B-RET transformation in lung adenocarcinoma. Lung Cancer. 2013;82:44-50.

\section{Submit your next manuscript to BioMed Central and take full advantage of:}

- Convenient online submission

- Thorough peer review

- No space constraints or color figure charges

- Immediate publication on acceptance

- Inclusion in PubMed, CAS, Scopus and Google Scholar

- Research which is freely available for redistribution

Submit your manuscript at www.biomedcentral.com/submit 